\title{
Basal Ganglia Glucose Utilization after Recent Precentral Ablation in the Monkey
}

\author{
George W. Dauth, PhD, Sid Gilman, MD, Kirk A. Frey, MD, PhD, and John B. Penney, Jr, MD
}

In the macaque monkey, unilateral ablation of areas 4 and 6 of Brodmann results initially in a significant decrease of glucose metabolic activity in the ipsilateral caudate nucleus, putamen, globus pallidus, substantia nigra, and subthalamic nucleus. The contralateral hemisphere shows nonsignificant but consistently decreased activity in the caudate nucleus, putamen, and globus pallidus. Cerebral blood flow is decreased in the same pattern as the glucose metabolic activity. The change in glucose metabolic activity results from loss of neurons known to project directly from the cerebral cortex to the basal ganglia and also from indirect effects (diaschisis) in basal ganglia structures that do not receive connections from the cerebral cortex.

Dauth GW, Gilman S, Frey KA, Penney JB Jr: Basal ganglia glucose utilization after recent precentral ablation in the monkey. Ann Neurol 17:431-438, 1985

Major sudden injury to the precentral or postcentral region of the cerebral cortex in the adult human leads to a well-known sequence of events affecting the motor system. The immediate result is paralysis of musculature on the contralateral side of the body. With time, partial recovery from the paralysis often occurs, together with the development of limb hypertonia and reflex hyperactivity. The degree of recovery is variable and little is known about the structures or processes underlying the initial paralysis or the restoration of motor function with time [11]. To study some of these factors, we have used a primate model of hemiplegia produced by unilateral removal of Brodmann's cerebral cortical areas 4 and 6 . This provides an excellent model of human hemiplegia, severe initially and lessening with time. In this communication we report the changes in glucose metabolic activity found in the basal ganglia during the initial phase of hemiplegia after cerebral cortical ablation. Preliminary results have been published $[4,5,9]$.

\footnotetext{
Methods

Thirteen Macaca fascicularis monkeys weighing 2.5 to $3.5 \mathrm{~kg}$ were used, including 6 unoperated controls and 7 with unilateral ablation of cerebral cortical areas 4 and 6 of Brodmann. The animals were examined neurologically before the operative procedure. Dexamethasone $(1 \mathrm{mg} / \mathrm{kg})$ and chloramphenicol sodium succinate $(25 \mathrm{mg} / \mathrm{kg}$ ) were injected subcutaneously daily for 2 days before and 3 days after surgery.

The animals were anesthetized with injections of $30 \mathrm{mg}$ of ketamine hydrochloride intramuscularly followed by $35 \mathrm{mg} /$ $\mathrm{kg}$ of sodium pentobarbital intraperitoneally. The operation
}

was performed using aseptic techniques. A bone flap was turned to expose the left hemisphere. The dura mater was reflected, and the rolandic and sylvian fissures and superior and inferior precentral sulci were used as landmarks for the ablation. Areas 4 and 6 of the cerebral cortex were ablated by subpial aspiration with much of the pia and blood supply left intact [11]. We removed the gray matter from the depths of the sulci. The ablation included about $2 \mathrm{~mm}$ of cortex on the medial surface of the hemisphere. After hemostasis was complete, the dura mater was closed with fine silk sutures, the bone flap was replaced, and the wound was closed in layers.

The animals were housed in separate cages, which permitted observation of their general behavior. Seven days after the ablation, we examined each animal neurologically [11] and ensured that they could undergo deoxyglucose incorporation. Dehydrated or lethargic animals were not used. The animal was strapped to a table and, under local anesthesia with lidocaine, arterial and venous catheters were placed in the right femoral artery and vein. The animal was restrained in a chair with straps around the neck, chest, pelvis, wrists, and ankles and then left in a quier room for 1 hour. We did not interfere with the animal's vision or hearing.

Deoxyglucose autoradiography was performed as described by Sokoloff and associates [29]. A bolus of $100 \mu \mathrm{Ci} /$ $\mathrm{kg}$ of $\left[{ }^{14} \mathrm{C}\right] 2$-deoxyglucose in $1 \mathrm{ml}$ of saline was injected through the venous catheter followed by a saline flush. Timed arterial blood samples were taken over the next 45 minutes. The last sample was taken at 45 minutes and then the animal was killed with $150 \mathrm{mg}$ of pentobarbital administered intravenously followed by $6 \mathrm{ml}$ of a saturated solution of potassium chloride. The brain and spinal cord were removed rapidly and frozen in isopentane cooled with dry ice.
From the Department of Neurology, The University of Michigan, Ann Arbor, MI 48109.

Received Jun 15, 1984, and in revised form Nov 19. Accepted for publication Nov 25, 1984.
Address reprint requests to $\mathrm{Dr}$ Dauth, The University of Michigan, Department of Neurology, Neuroscience Laboratory Building, 1103 East Huron St, Ann Arbor, MI 48104. 
The brain was removed with the head immobilized in a stereotaxic head holder. With a knife mounted on the head holder, the brain was sliced stereotaxically into two sections of approximately equal volume. The tissue was coated with Lipshaw M-1 embedding matrix to retard dehydration during storage. The brain and plasma samples were stored at $-70^{\circ} \mathrm{C}$ until used. The plasma glucose levels were measured with a Beckman glucose analyzer and the plasma ${ }^{14} \mathrm{C}$ levels were measured with a Beckman LS9000 scintillation counter. The brain was sectioned at $20 \mu \mathrm{m}$ in a Lipshaw $1800 \mathrm{~N}$ motorized cryostat. Three serial sections were taken every $400 \mu \mathrm{m}$ throughout the brain for autoradiography. The sections were placed on coverslips and dried rapidly on a hot plate. The sections, along with calibrated ${ }^{14} \mathrm{C}$ standards, were placed on Kodak SB-5 $\mathrm{x}$-ray film in a cassette, exposed for 6 days, and then developed.

The autoradiograms were analyzed with a spot densitometer interfaced to a microcomputer [3]. The computer was programmed to read the film density and convert to glucose utilization rates [29]. The lumped and rate constants used were those of Kennedy and co-workers [14]. The glucose utilization rates were determined for each structure every $400 \mu \mathrm{m}$ throughout the brain. At each level the measurement consisted of the average of multiple readings from the three serial sections at that level. Some sections, about $2 \%$ of the total, were cut excessively thick or thin. Data from these sections were not included in the analysis. Each structure was measured in the left and right hemispheres separately. The final values used in the statistical analysis consisted of the average from all levels for a particular structure. A two-way repeated measures ANOVA was used on the data for each structure to determine left-right and group differences. The significant differences reported are based on tests of simple main effects, which were determined by significant group by side interaction effects [32].

Two 20- $\mu \mathrm{m}$ serial sections adjacent to those used for autoradiography were processed for histological study. These were thaw-mounted onto subbed slides and postfixed over paraformaldehyde vapor at $38^{\circ} \mathrm{C}$ for 3 hours. Before staining, the slides were washed in distilled water for 3 minutes. One section then was stained with cresyl violet [19] and the other with a $0.3 \%$ solution of osmium tetroxide in distilled water until the myelin was well stained. Sections were then washed, dehydrated through alcohols and xylene, enclosed in Permount (Fisher Scientific), coverslipped, and examined under a microscope.

A single animal with ablation of areas 4 and 6 of Brodmann of 1 week's duration was used to study cerebral blood flow [25]. The animal was injected with $50 \mu \mathrm{Ci} / \mathrm{kg}$ of $\left[{ }^{14} \mathrm{C}\right]$ iodoantipyrine at a constant rate over 1 minute. The animal was killed and the brain was removed and processed as described.

\section{Results}

\section{Clinical Effects of Precentral Cortical Ablation}

On examination on the seventh day after ablation of the left areas 4 and 6 , a typical animal sat in its cage with the left limbs flexed, the right arm three-quarters extended, and the right leg three-quarters extended, abducted at the hip, and externally rotated. The left hand and foot usually oriented to the cage surfaces so that the fingers and toes curled around the wire or bars. The right hand and foot did not orient to these surfaces and the digits were loosely flexed, touching the surfaces lightly. The animal walked using both legs, but often the right leg flexed more than the left during stepping, with smaller-amplitude strides, and frequently lagged behind the left. When the animal stood still, the right leg often was more extended and externally rotated than the left. The animal reached for a stick using the left hand, grasped it deftly, and pulled it toward its mouth. The right arm usually did not reach out, but at times the arm extended after the left hand had grasped the stick. The right hand then touched the stick lightly, but the fingers failed to orient to the surface. The animal neither supported its body weight with the right arm nor attempted to walk using that extremity.

When the animal was suspended from the chest in the upright or inverted position, the right limbs hung passively in extension and the left limbs were flexed and adducted, although all four limbs showed intermittent struggling movements. When the animal was blindfolded, the left limbs showed a placing reaction in response to light cutaneous contact. The right limbs did not place in response to contact with either the right or left limbs. When the animal was tilted laterally, there was a poor abduction response with the right shoulder and a rapid response with the left. Hopping responses were poor in the right limbs. When the animal was immobilized in a chair with straps about the neck, chest, and pelvis, the right limbs hung threequarters extended, failing to contact the surfaces of the chair, and the left limbs remained flexed with the hand and foot grasping the surfaces of the chair. The right hand and foot showed no response to light contact to the palm or sole, whereas the left hand and foot responded immediately with avoiding or grasping reactions. On passive manipulation the right limbs showed less than normal resistance, with only a barely detectable plastic resistance appearing in the extensors and flexors of the elbow, knee, and ankle. Passive manipulation of the other joints produced essentially no resistance. The left limbs showed natural resistance to manipulation. The deep tendon reflexes were diminished in amplitude in the right limbs when compared with the left. The patellar reflex on the right was slower than on the left and was pendular.

\section{Histological Results}

The histological patterns were similar in all cases. The lesions involved essentially all of the agranular isocortex and extended deeply into the underlying white matter, sparing the deep nuclei (Fig 1A). The lesions spared the extreme medial portion of the prefrontal gyrus and the depths of the superior precentral sulcus. 


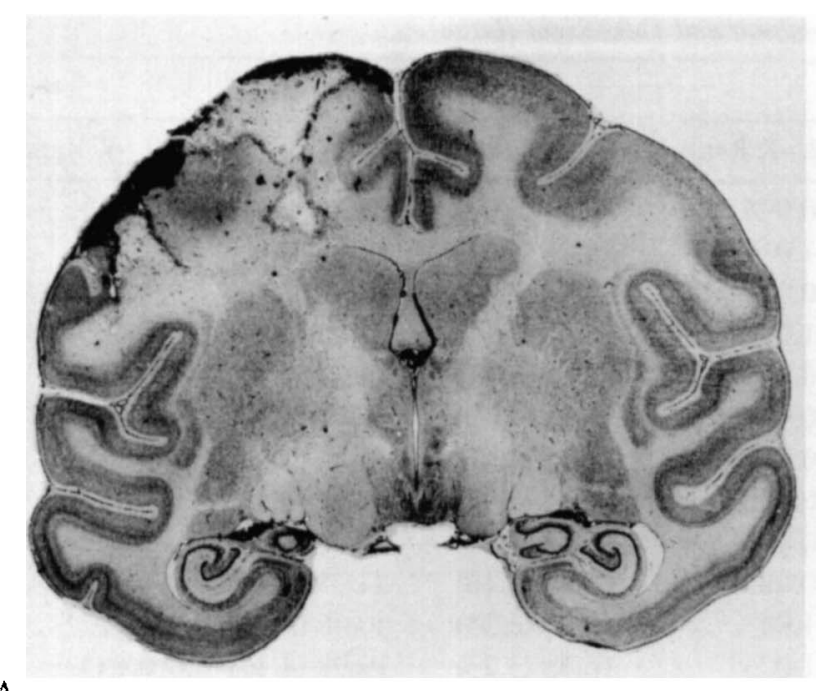

A

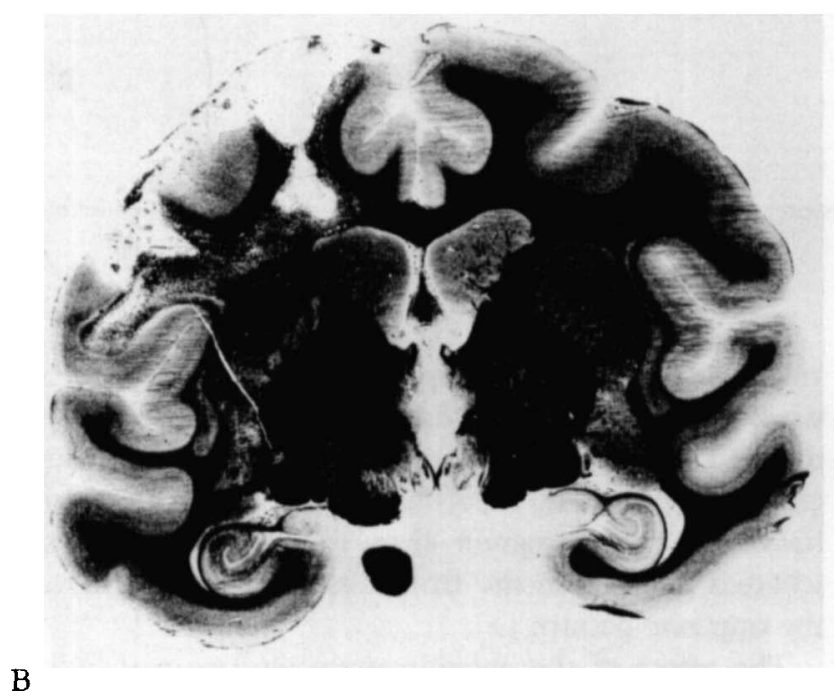

Fig 1. Coronal sections through the lesion of a monkey 1 week after ablation of cerebral cortical areas 4 and 6. (A) Cresyl violet-stained section showing the extent of the lesion in agranular isocortex, but sparing the most medial part of the precentral gyrus and all deep gray matter nuclei. (B) Osmium-stained section showing myelin pallor indicating edema in the white matter beneath the lesion. Edema was not present in the deep nuclei.

Granular isocortex was uninvolved. An infiltrate of mononuclear and a few polymorphonuclear inflammatory cells at the edges of the lesion extended into the subarachnoid space for several millimeters. In the white matter underlying the lesion were perivascular cuffs of mononuclear cells, a mild mononuclear infiltrate, and a number of reactive astrocytes. Slight perivascular and tissue infiltrates of mononuclear cells were found in the putamen and the ventral anterior, ventral lateral, and centromedian nuclei of the thalamus and the rostral portion of the red nucleus. Small infiltrates of mononuclear cells and some increase in numbers of astrocytes were found in the portions of the internal capsule, crus cerebri, and pontine white matter through which corticospinal fibers had passed, and in the medullary pyramid and contralateral lateral column of the spinal cord.

Osmium-stained sections (Fig 1B) revealed myelin pallor, suggesting edema in the white matter underlying the lesion. The edema extended almost to the body of the caudate nucleus but spared it in all but one case. Myelin staining was pale along the course of the corticospinal tract in the internal capsule, crus cerebri, pons, medullary pyramid, and lateral column. In one animal the myelin pallor extended into the body of the caudate nucleus. This animal had slight subfalcial herniation.

\section{Local Cerebral Metabolic Rates for Glucose}

The average local cerebral metabolic rate for glucose (LCMRGlc) for 6 unoperated control animals showed no significant differences between homologous structures in the left and right hemispheres (Table). Analysis of data from 6 animals with ablation of cerebral cortical areas 4 and 6 in the left hemisphere revealed significant differences in LCMRGic within the basal ganglia between the two hemispheres (Fig 2, Table). No significant left-right differences were found for the septal region, medial geniculate nucleus, or lateral geniculate nucleus.

In both hemispheres of the control animals the caudal portions of both the caudate nucleus and the putamen had a higher LCMRGlc than the rostral portions. There was a similar relationship in the right hemisphere of the lesioned animals, but not in the left hemisphere (Table). The left-right ratios for the caudate nucleus in a representative lesioned animal started in the rostral portion at a value close to 1.00 and gradually decreased in the more caudal portions until the level of the anterior commissure (Fig 3). Caudal to the anterior commissure the left-right ratios remained depressed at a more or less constant level. Similar results along the rostral-caudal axis were observed for the putamen, but not for the other structures listed in the Table. Because of these observations, in the present analysis the caudate nucleus and putamen were divided into rostral and caudal portions, the rostral portion consisting of the segment rostral to the anterior commissure and the caudal portion extending from the anterior commissure to the level of the red nucleus.

Comparisons between ablated and control groups revealed a significant decrease in LCMRGlc in the left putamen, pallidum, and substantia nigra (Table). The decrease in the left caudate nucleus was not statistically significant in the comparison between groups, probably because of the large variance between groups. The decreases in the left pallidum were significant, although the absolute magnitude of the decrease was small (Table). None of the comparisons between ab- 
Mean (SEM) Local Cerebral Metabolic Rates for Glucose in $\mu \mathrm{M} / 100 \mathrm{gm} / \mathrm{min}$ and Left-Right Ratios

\begin{tabular}{|c|c|c|c|c|c|c|}
\hline \multirow[b]{2}{*}{ Location } & \multicolumn{3}{|c|}{ Control } & \multicolumn{3}{|c|}{ Lesion } \\
\hline & Left & Right & L-R Ratio & Left & Right & L-R Ratio \\
\hline Rostral caudate & $30.17(2.61)$ & $30.35(2.59)$ & 0.994 & $25.43(2.92)$ & $27.78(2.93)^{a}$ & 0.915 \\
\hline Caudal caudate & $35.00(2.70)$ & $34.47(2.58)$ & 1.015 & $26.23(3.63)$ & $32.45(4.25)^{a}$ & 0.808 \\
\hline Rostral putamen ${ }^{b}$ & $31.70(2.78)$ & $31.78(2.83)$ & 0.997 & $20.62(2.56)$ & $28.82(2.95)^{a}$ & 0.715 \\
\hline Caudal putamen ${ }^{c}$ & $34.40(2.27)$ & $34.05(2.26)$ & 1.010 & $19.52(2.65)$ & $31.22(3.98)^{a}$ & 0.625 \\
\hline Pallidum internal ${ }^{b}$ & $16.28(1.08)$ & $16.73(1.20)$ & 0.973 & $11.78(1.51)$ & $14.60(1.82)^{\mathrm{a}}$ & 0.807 \\
\hline Pallidum external ${ }^{b}$ & $16.38(0.95)$ & $16.47(1.13)$ & 0.995 & $11.68(1.79)$ & $14.17(1.97)^{\mathrm{a}}$ & 0.824 \\
\hline Septum & $18.03(1.30)$ & $18.12(1.47)$ & 0.995 & $14.95(1.43)$ & $15.27(1.40)$ & 0.979 \\
\hline Subthalamic nucleus & $30.87(1.47)$ & $31.58(1.94)$ & 0.978 & $27.63(3.97)$ & $31.27(4.47)^{\mathrm{a}}$ & 0.884 \\
\hline Red nucleus & $27.52(1.32)$ & $27.72(1.41)$ & 0.993 & $20.02(3.58)$ & $30.72(5.97)^{\mathrm{a}}$ & 0.652 \\
\hline Lateral geniculate nucleus & $24.48(1.46)$ & $24.93(1.65)$ & 0.982 & $24.83(5.18)$ & $24.95(5.17)$ & 0.995 \\
\hline Medial geniculate nucleus & $42.52(2.56)$ & $40.75(2.31)$ & 1.043 & $42.70(6.35)$ & $46.08(7.98)$ & $0.927^{\mathrm{d}}$ \\
\hline Substantia nigra reticulata ${ }^{b}$ & $20.42(1.13)$ & $19.96(0.96)$ & $1.023^{d}$ & $16.88(3.23)$ & $19.98(4.36)^{\mathrm{c}}$ & 0.845 \\
\hline Substantia nigra compacta ${ }^{b}$ & $27.48(1.36)$ & $28.12(1.55)$ & $0.977^{d}$ & $25.02(3.80)$ & $28.85(5.16)^{\mathrm{c}}$ & 0.867 \\
\hline
\end{tabular}

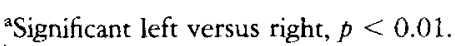

'Significant control versus ablated left hemisphere, $p<0.05$.

'Significant control versus ablated left hemisphere, $p<0.01$.

The data are based on 5 rather than 6 animals.

'Significant left versus right, $p<0.05$.

$\mathrm{L}=$ left; $\mathrm{R}=$ right; rostral $=$ measurements rostral to the anterior commissure; caudal = measurements between the anterior commissure and the ted nucleus. Further explanation is provided in the text and Figure 3.

lated and control groups for the structures in the right hemispheres showed significant differences, but the data indicate a trend toward reduced activity in the basal ganglia of the right hemisphere.

The effect of an ablative procedure in the cerebral cortex upon the blood supply of underlying subcortical structures is of concern as some of the changes in metabolic activity could result from interruption of blood flow. We have performed a single experiment to assess the effect of the lesion on blood flow as measured with $\left[{ }^{14} \mathrm{C}\right]$ iodoantipyrene. The results reveal a pattern of diminished blood flow that replicates closely the pattern of altered metabolic activity demonstrated with 2-deoxyglucose (Fig 4).

\section{Discussion}

The present study has demonstrated that ablation of areas 4 and 6 of Brodmann in the left hemisphere of the macaque monkey results in a decrease in glucose metabolic activity 1 week after the lesion in the left caudate nucleus, puramen, globus pallidus, substantia nigra, and subthalamic nucleus. No decrease occurs in the septum or in the medial and lateral geniculate nuclei. The right hemisphere shows nonsignificant but consistently decreased activity in the caudate nucleus, putamen, and globus pallidus. These results are similar to those of Deuel and Collins [6, 7], who studied monkeys with unilateral lesions of the frontal association cortex. However, their data suggest that the caudate nucleus may be more affected than the putamen [6], whereas we found the opposite. The results of their work and ours are consistent with the known distribution of cerebral cortical projections to the caudate nucleus and putamen. Neurons in area 4 project more heavily to the putamen than the caudate nucleus, whereas neurons in the frontal association areas have the opposite pattern [1].

The effect of the ablation upon glucose metabolic activity is most pronounced in the putamen, followed by the caudate nucleus and then the remaining structures, which are much less affected. The ablation results in not only decreased activity but also increased variability of activity in the affected structures. This increase in variability, coupled with the variability inherent in the deoxyglucose technique, leads to some difficulty in statistical interpretation of the results, especially in the case of the caudate nucleus. Kuhl and co-workers [15] experienced similar variability in their positron emission tomography studies of patients with stroke. They normalized their data to a structure that did not appear to be affected by the stroke. This technique only provides a certain degree of consistency from case to case and does not solve the potential problem of changes in the lumped or rate constants with diseased or lesioned tissue. We used a similar technique by normalizing the data for the basal ganglia to the lateral geniculate nucleus, a structure not affected by the lesion. We found no significant difference between the normalized and nonnormalized data and chose to present them in the nonnormalized form. 

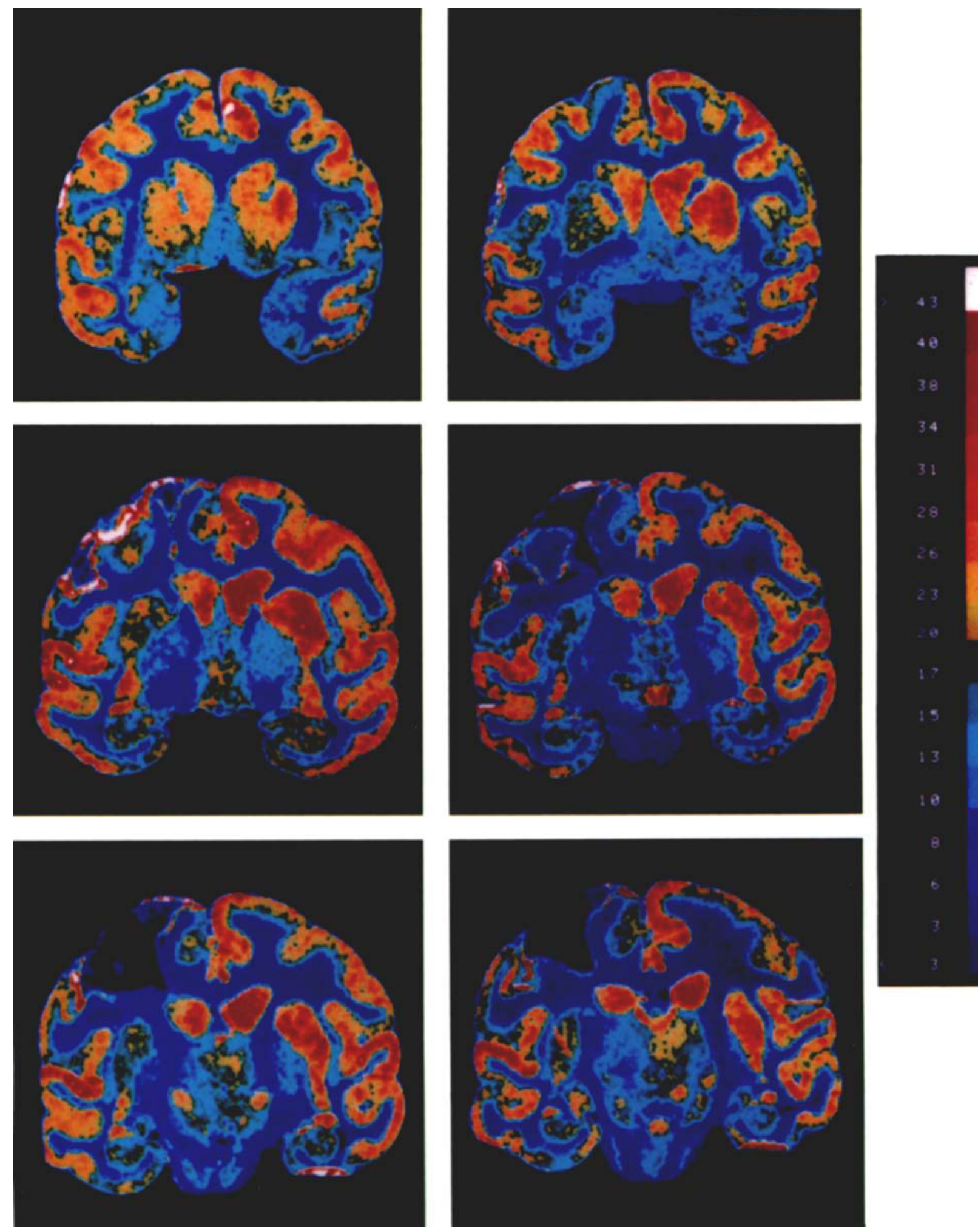

Fig 2. Computer-processed quantitative autoradiograms of a monkey brain 1 week after ablation of cerebral cortical areas 4 and 6. The color bar on the right is coded for cerebral glucose utilization rates. 


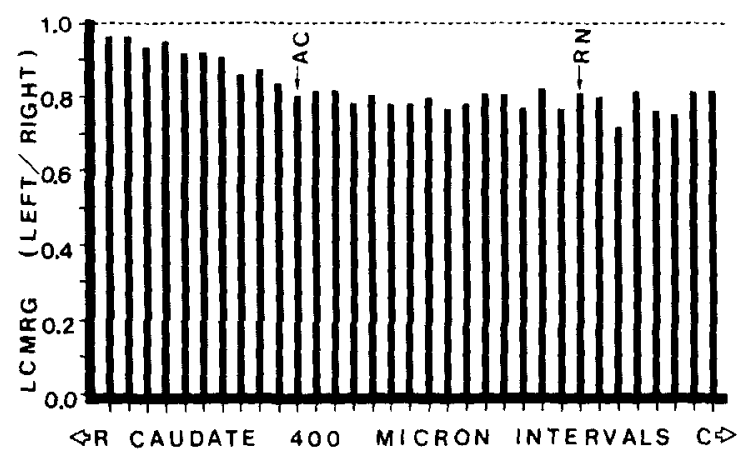

Fig 3. Histogram sbowing left-right ratios of local cerebral metabolic rates for glucose (LCMRG) in the caudate nucleus from 1 animal 1 week after unilateral ablation of cerebral cortical areas 4 and 6 . The ratios are from rostral $(R)$ to caudal $(C)$ at 400- $\mu$ intervals. $(\mathrm{AC}=$ anterior commissure $\mathrm{RN}=\mathrm{red}$ nucleus.)

The comparisons of activity in the left and right hemispheres for the caudate nucleus in animals with cerebral ablacions showed highly significant differences, as did the interaction between groups and hemispheres. The comparisons between control and lesion groups for the caudate nucleus of the left (lesioned) hemisphere did not show significant differences, possi- bly because of the large variability. Nevertheless, other information indicates that the changes in the caudate nucleus are important. The left-right ratios for the caudate nucleus show a distinctive pattern from the most rostral section to well caudal to the red nucleus (Fig 3) in virtually all of the lesioned animals. Apart from the most rostral sections, the left caudate nucleus shows a lower glucose utilization rate than the right caudate nucleus. A similar pattern occurs in the putamen and globus pallidus and, to a lesser extent, in the remaining structures with significant left-right differences.

Some of the changes in glucose metabolic activity in the basal ganglia may result directly from loss of activity in neurons known to project from areas 4 and 6 into the basal ganglia. Thus, topographically organized projections extend from the lesioned area to the putamen and caudate nucleus, although the density of projections from areas 4 and 6 to the putamen are greater

Fig 4. Computer-processed $\left({ }^{14} \mathrm{C}\right)$ iodoantipyrine autoradiograms from an animal 1 week after unilateral ablation of cerebral cortical areas 4 and 6 . The autoradiograms are not quantitative and are presented only to illustrate the relative distribution of blood flow. The relative concentrations are from red (bighest) to violet (lowest).
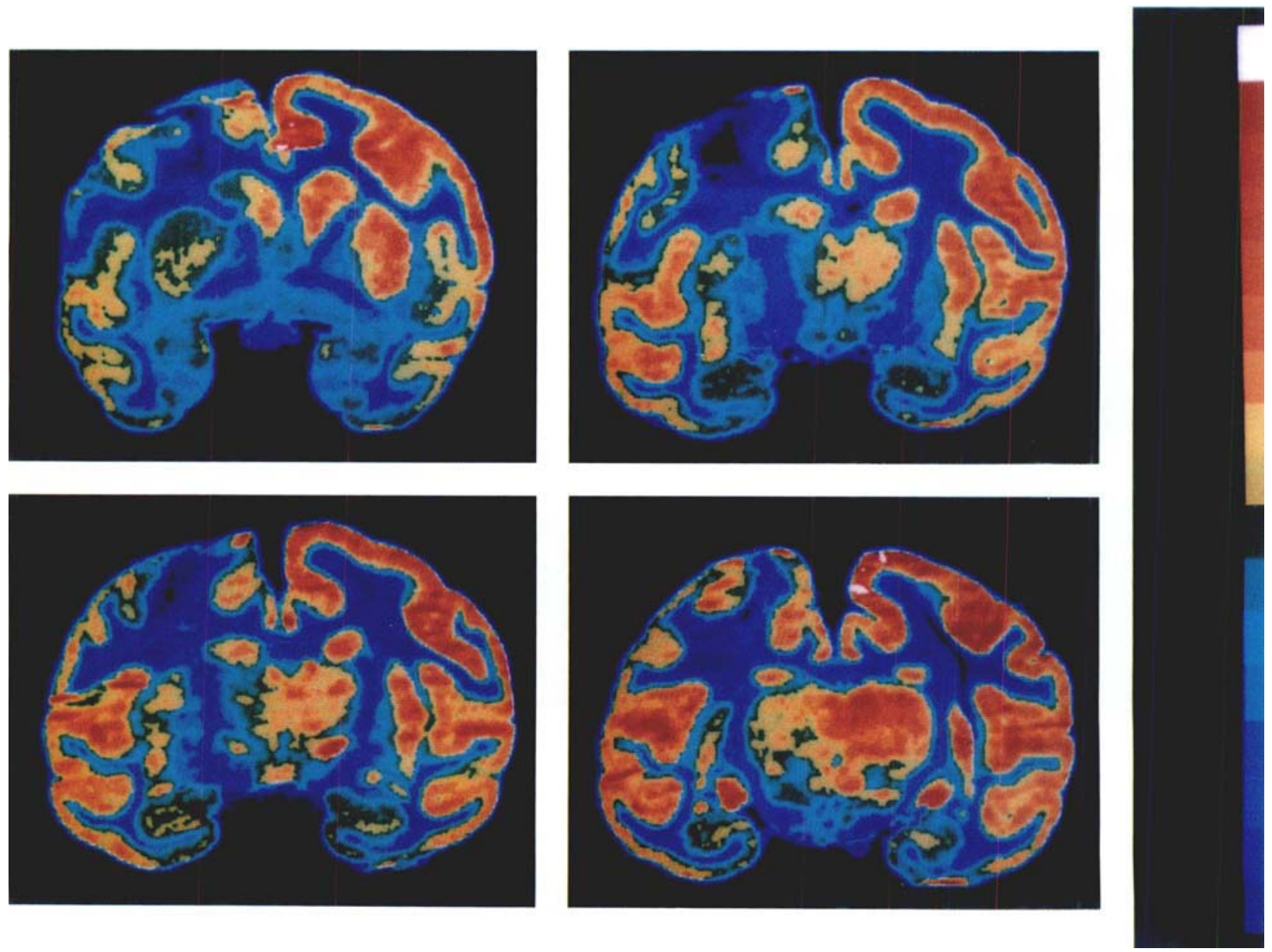
than those to the caudate nucleus $\{16,17\}$. Neurons in the frontal lobe project to the anterior part of the head of the caudate nucleus and to the precommissural part of the putamen. Projections occur bilaterally also to a large anteroposterior division of the putamen in a somatotopically organized pattern with fibers from the cortical leg and tail areas terminating most rostrodorsally and those from the face area terminating most caudoventrally [16].

Degeneration of corticofugal fibers projecting directly to the substantia nigra and subthalamic nucleus may contribute to the decreased metabolic activity in these structures. The existence of a corticonigral projection has been doubted in the past $[22,23]$, but recent studies have revealed some connections from the prefrontal cortex to the substantia nigra $[2,12$, 30]. In addition, a projection has been found from areas 6 and 9 in the monkey to the pars compacta of the substantia nigra [17]. A topographical projection has been described in monkeys extending from cerebral cortical areas 4 and 6 to the ipsilateral subthalamic nucleus [13].

Because cerebral cortical projections extending directly to the globus pallidus have not been described, the decreased metabolic activity in the pallidum observed in the present study cannot be attributed to loss of direct corticofugal projections. Rather, the decreased pallidal activity must result from transsynaptic effects. Loss of the excitatory projections from the cerebral cortex to the caudate nucleus and putamen evidently alters the activity of neostriatal neurons that project to the globus pallidus [24]. In addition, altered activity in the subthalamic nucleus from loss of corticofugal terminals may affect the pallidum. Similar transsynaptic effects, termed diaschisis, have been observed in the cerebellar hemisphere opposite a lesion in the cerebral cortex $[18,20]$.

Our data consistently show a small reduction in metabolic activity in the right basal ganglia. This finding is consistent with anatomical studies demonstrating direct projections from areas of the cerebral cortex to the contralateral basal ganglia $[8,16,17]$. Our studies have shown that several subcortical structures apart from the basal ganglia are also affected by the lesion, including the left thalamus, red nucleus, pons, and the right cerebellar hemisphere $[4,5,9,10]$.

The changes in glucose metabolic activity within the central nervous system found in this study are compatible with the clinical situation in which loss of function of the precentral region of the cerebral cortex results initially in an essentially complete hemiplegia [11]. Neurons arising in the precentral cortex make extensive connections through the basal ganglia, brainstem, and spinal cord. Many of these neurons utilize glutamate or aspartate as a neurotransmitter and are strongly excitatory in their synaptic effects [33]. Thus, the initial hemiplegia may be viewed as a result of the withdrawal of strongly excitatory synaptic connections, both from loss of direct excitatory glutamatergic connections and also from transsynaptic effects.

The changes in glucose metabolic activity in animals with hemiplegia from cerebral cortical ablation do not reflect differences in the relative use of the limbs on the two sides of the body. During deoxygluose incorporation we maintained our animals strapped in a chair with all four limbs firmly secured to prevent asymmetrical levels of sensory input from the two sides of the body. Moreover, we have examined metabolic activity in the cuneate nuclei and have found no difference between the two sides [9]. Greater sensory input from one side should have been detected as an asymmetry in metabolic rate at the level of the cuneate nucleus.

Decreased metabolic activity within the basal ganglia in animals with precentral cortical ablation could result from a decrease of local cerebral blood flow if blood vessels penetrating into the basal ganglia were injured. This possibility is unlikely for several reasons. First, deoxyglucose uptake depends primarily on cellular [31] and synaptic terminal [26-28] activity; it is not limited by blood flow, either in cases of physiological variation or in many kinds of pathological alterations of neuronal function [21]. Second, our histological analysis of sections adjacent to those used for autoradiography revealed no evidence of tissue ischemia. Finally, a study of cerebral blood flow in one animal 1 week after ablation of areas 4 and 6 gave no evidence that perfusion was decreased to critical levels. The pattern of decreased blood flow was strikingly similar to that of decreased glucose metabolic activity, even in the cerebellum and brainstem, which are remote from the site of the lesion and receive their blood supply from the posterior circulation [9]. We conclude that the alterations in deoxyglucose uptake reflect changes brought about by the loss in function of neural connections and subsequent transsynaptic effects and that changes in blood flow follow the pattern of decreased metabolic activity.

Glucose urilization rates in the present study were measured using techniques similar to those reported by Kennedy and associates [14]. The lumped and rate constants developed by those investigators were used in the present study. The glucose utilization rates for our control animals were generally lower than those reported by Kennedy and associates [14]. There may be several reasons for this difference. First, we used $M$. fascicularis, whereas they used M. mulatta; there may be differences in cerebral glucose metabolic rates between the two species. Second, we used a local anesthetic for insertion of the catheters, whereas they used general anesthesia. Third, we may have handled tissues differently. In our studies the tissues from both control and lesioned animals were treated in the same manner and our data appear to be internally consistent.

Dauth et al: Basal Ganglia Glucose Utilization 
Supported in part by Grant NS 15655 from the National Institute of Neurological and Communicative Disorders and Stroke and Grant R-291-81 from the United Cerebral Palsy Educational and Research Foundation, Inc. K. A. F. was a trainee on Grant GM 07863 from the National Institutes of Health.

\section{References}

1. Brodal A: Neurological Anatomy. Third edition. New York, Oxford University Press, 1981

2. Bunney BS, Aghajanian GK: The precise localization of nigral afferents in the rat as determined by retrograde tracing technique. Brain Res 117:423-435, 1976

3. Dauth GW, Frey KA, Gilman S: A densitometer for quantitative autoradiography. J Neurosci Meth 9:243-251, 1983

4. Dauth G, Gilman S, Frey K, Penney J: $\left[{ }^{1 / 4} \mathrm{C}\right\}$-2-deoxyglucose uptake in monkeys with hypotonic hemiplegia after precentral or postcentral lesions. Neurology (NY) 30:407, 1980

5. Dauth $\mathrm{G}$, Gilman $\mathrm{S}$, Frey $\mathrm{K}$, et al: $\left[{ }^{14} \mathrm{C}\right]$-2-deoxyglucose uptake in monkeys with hypotonic hemiplegia after precentral cortical ablation. Neurosci Abstr 5:367, 1979

6. Deuel RK, Collins RC: Recovery from unilateral neglect. Exp Neurol 81:733-748, 1983

7. Deuel RK, Collins RC: The functional anatomy of frontal lobe neglect in the monkey: behavioral and quantitative 2deoxyglucose studies. Ann Neurol 15:521-529, 1984

8. Fallon JH, Ziegler BTS: The crossed cortico-caudate projection in the rhesus monkey. Neurosci Lett 15:29-32, 1979

9. Gilman S, Dauth GW, Frey KA, et al: Basal ganglia glucose metabolic and single neural unit activity in experimental hemiplegia. Int J Neurol (in press, 1985)

10. Gilman S, Dauth G, Frey K, Penney J: Asymmetries of glucose utilization in the basal ganglia of hypotonic and hypertonic hemiplegic monkeys after precentral cortical ablation. Neurology (NY) 31:150, 1981

11. Gilman S, Lieberman JS, Marco LA: Spinal mechanisms underlying the effects of unilateral ablation of areas 4 and 6 in monkeys. Brain 97:49-64, 1974

12. Grofová I: Extrinsic connections of the neostriatum. In Divac I, Öberg RE (eds): The Neostriatum. Oxford, Pergamon, 1979, pp 37-51

13. Hartmann-von Monakow K, Akert K, Künzle $\mathrm{H}$ : Projections of the precentral motor cortex and other cortical areas of the frontal lobe to the subthalamic nucleus in the monkey. Exp Brain Res 33:395-403, 1978

14. Kennedy C, Sakurada O, Shinohara M, et al: Local cerebral glucose utilization in the normal conscious macaque monkey. Ann Neurol 4:293-301, 1978

15. Kuhl DE, Phelps ME, Kowell AP, et al: Effects of stroke on local cerebral metabolism and perfusion: mapping by emission computed tomography of ${ }^{18} \mathrm{FDG}$ and ${ }^{13} \mathrm{NH}_{3}$. Ann Neurol $8: 47-60,1980$

16. Künzle $\mathrm{H}$ : Bilateral projections from precentral motor cortex to the putamen and other parts of the basal ganglia. An autoradio- graphic study in Macaca fascicularis. Brain Res 88:195-209, 1975

17. Künzle H: An autoradiographic analysis of the efferent connections from premotor and adjacent prefrontal regions (areas $6 \&$ 9) in Macaca fascicularis. Brain Behav Evol 15:185-234, 1978

18. Kushner M, Alavi A, Reivich M, et al: Contralateral cerebellar hypometabolism following cerebral insult: a positron emission tomographic study. Ann Neurol 15:425-434, 1984

19. Luna LG: Manual of histologic staining methods of the Armed Forces Institute of Pathology. Third edition. New York, McGraw-Hill, 1968

20. Martin WRW, Raichle ME: Cerebellar blood flow and metabolism in cerebral hemisphere infarction. Ann Neurol 14:168176,1983

21. Rapin JR, Lageron A, Le Poncin-Lafitte M: Deoxyglucose uptake in pathological conditions. Eur Neurol 20:146-151, 1981

22. Rinvik E: The cortico-nigral projection in the car. An experimental study with silver impregnation methods. J Comp Neurol 126:241-254, 1966

23. Rinvik E, Walberg F: Is there a cortico-nigral tract? A comment based on experimental electronmicroscopic observations in the cat. Brain Res 14:742-744, 1969

24. Royce GJ, Laine EJ: Efferent connections of the caudate nucleus, including cortical projections of the striatum and other basal ganglia: an autoradiographic and horseradish peroxidase investigation in the cat. J Comp Neurol 226:28-49, 1984

25. Sakurada O, Kennedy C, Jehle J, et al: Measurement of local cerebral blood flow with iodo $\left[{ }^{14} \mathrm{C}\right]$ antipyrine. Am J Physiol 234:H59-H66, 1978

26. Schwartz WJ, Sharp FR: Autoradiographic maps of tegional brain glucose consumption in resting, awake rats using $\left[^{-14} \mathrm{C}\right]-2-$ deoxyglucose. J Comp Neurol 177:335-360, 1978

27. Schwartz WJ, Smith CB, Davidsen L, et al: Metabolic mapping of functional activity in the hypothalamoneurohypophysial system of the rat. Science 205:723-725, 1979

28. Sokoloff L: Relation berween physiological function and energy metabolism in the central nervous system. J Neurochem 29:1326, 1977

29. Sokoloff L, Reivich M, Kennedy $C$, et al: The $\left[{ }^{14} \mathrm{C}\right]-$ deaxyglucose method for the measurement of local cerebral glucose utilization: theory, procedure and normal values in the conscious and anesthetized albino rat. J Neurochem 28:897916, 1977

30. Tulloch IF, Arbuthnott GW, Wright AK: Topographical organization of the striatonigral pathway revealed by anterograde and retrograde neuroanatomical tracing techniques. J Anat 127: 425-441, 1978

31. Wagner HJ, Hoffmann KP, Zwerger H: Layer-specific labelling of cat visual cortex after stimulation with visual noise: a $\left.{ }^{3} \mathrm{H}\right] 2-$ deoxy-d-glucose study. Brain Res 224:31-43, 1981

32. Winer BJ: Statistical Principles in Experimental Design. New York, McGraw-Hill, 1967

33. Young AB, Penney JB, Dauth GW, et al: Glutamate or aspartate as a possible neurotransmitter of cerebral corticofugal fibers in the monkey. Neurology (Cleveland) 33:1513-1516, 1983 